\title{
Low-loss and bendable THz fiber with tailored dispersion
}

Nielsen, Kristian; Rasmussen, Henrik K.; Adam, Aurèle J. L.; Planken, Paul C.M.; Bang, Ole; Jepsen, Peter Uhd

Published in:

Proceedings of the Conference on Lasers and Electro-Optics

Publication date:

2009

Document Version

Publisher's PDF, also known as Version of record

Link back to DTU Orbit

Citation (APA):

Nielsen, K., Rasmussen, H. K., Adam, A. J. L., Planken, P. C. M., Bang, O., \& Jepsen, P. U. (2009). Low-loss and bendable $\mathrm{THz}$ fiber with tailored dispersion. In Proceedings of the Conference on Lasers and Electro-Optics (pp. 1-2). IEEE.

\section{General rights}

Copyright and moral rights for the publications made accessible in the public portal are retained by the authors and/or other copyright owners and it is a condition of accessing publications that users recognise and abide by the legal requirements associated with these rights.

- Users may download and print one copy of any publication from the public portal for the purpose of private study or research.

- You may not further distribute the material or use it for any profit-making activity or commercial gain

- You may freely distribute the URL identifying the publication in the public portal

If you believe that this document breaches copyright please contact us providing details, and we will remove access to the work immediately and investigate your claim. 


\title{
CThQ7.pdf
}

\section{Low-Loss and Bendable THz Fiber with Tailored Dispersion}

\author{
Kristian Nielsen, ${ }^{1}$ Ole Bang, ${ }^{1}$ Henrik K. Rasmussen, ${ }^{2}$ Auréle J. L. \\ Adam, ${ }^{3}$ Paul C. M. Planken, ${ }^{3}$ and Peter Uhd Jepsen ${ }^{1}$ \\ ${ }^{1}$ Technical University of Denmark, Department of Photonics Engineering, DK-2800 Kongens \\ Lyngby, Denmark \\ ${ }^{2}$ Technical University of Denmark, Department of Mechanical Engineering, DK-2800 Kongens \\ Lyngby, Denmark \\ ${ }^{3}$ Delft University of technology, Faculty of Applied Physics, Department of Imaging Science \\ and Technology, Lorentzweg 1, 2628 CJ Delft, the Netherlands \\ krini@fotonik.dtu.dk
}

\begin{abstract}
A polymer THz fiber made of Topas and having a Photonic Crystal Fiber structure is demonstrated. It has low broadband loss and the dispersion of the fiber can be tailored by adjusting the structural parameters.
\end{abstract}

(c) 2009 Optical Society of America

OCIS codes: (060.5295) Photonic Crystal Fibers; (260.3090) Infrared, far

In this paper we report on the properties of the first THz polymer photonic crystal fiber (PCF) made of Topas ${ }^{\circledR}$ cyclic olefin copolymers (COC). Topas has previously been used for microstructured optical fiber [3] but to our knowledge this is the first time Topas has been used for THz fiber. The reason for using Topas is that it has exceptionally low loss and flat dispersion in the $\mathrm{THz}$ range [1]. In this paper we report on the propagation loss and on the dispersion of the THz Topas PCF. The properties of the fiber have also been modeled numerically using the full vectorial software package COMSOL. From these numerical calculations the dispersion of the fiber has been calculated. The calculated dispersion profile is compared to the measured dispersion and shows excellent agrement.

The polymer fibers are made by using a draw tower. The preform structure is drilled into a solid $60 \mathrm{~mm}$ Topas rod and then drawn down to approximately $6 \mathrm{~mm}$ diameter THz fibers. The fibers are robust and easy to handle. The fiber can be cleaved and also bent around tight corners without a significant increase in loss. In general, handling of the fibers is made easier by their size and softness.
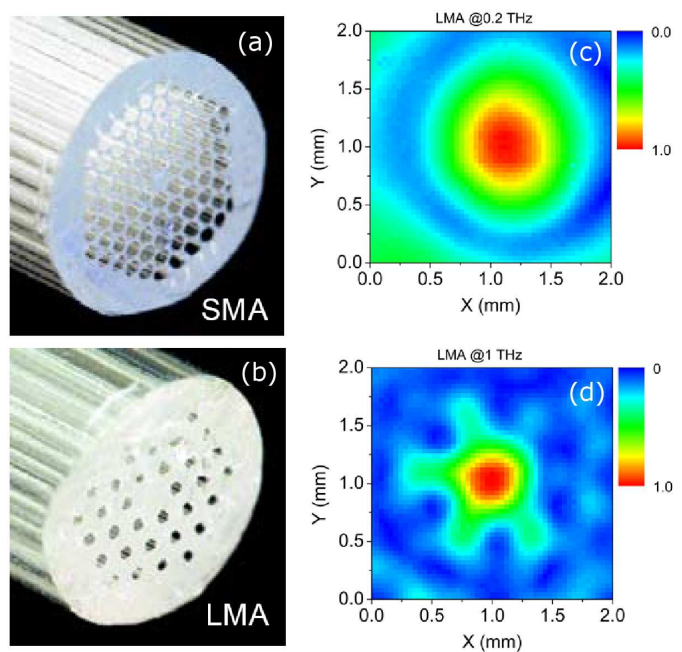

Fig. 1. On the left there are images of the two fiber presented in this paper. (a) is the SMA fiber and (b) is of the LMA fiber. On the right near-field experimental measurements of the mode profile of the LMA fiber are shown at two frequencies. (c) is at $0.2 \mathrm{THz}$ where the mode is loosely confined to the core and (d) is at $1 \mathrm{THz}$ where the mode is tightly confined to the core and the hexagonal profile of the fiber is clearly visible.

In this paper we demonstrate the design freedom with two fibers shown in Fig. 1 (a) and (b). The large mode area (LMA) fiber has a hexagonal lattice pitch of $560 \mu \mathrm{m}$ and a hole diameter of $250 \mu \mathrm{m}$ giving a hole diameter to pitch ratio of 0.45 . The small mode area (SMA) fiber has a pitch of $350 \mu \mathrm{m}$ and a hole diameter of $280 \mu \mathrm{m}$ giving a ratio of 0.8 . The small hole diameter to pitch ratio of the LMA fiber ensures that the fiber is practically single moded, while the high ratio of the SMA fiber makes it multi moded. 


\section{CThQ7.pdf}

The mode profile of the two fibers is also characterized experimentally by using near-field electro-optic sampling [2]. The images from the near-field sampling are shown together with images of the fibers in Fig. 1 (c) and (d).
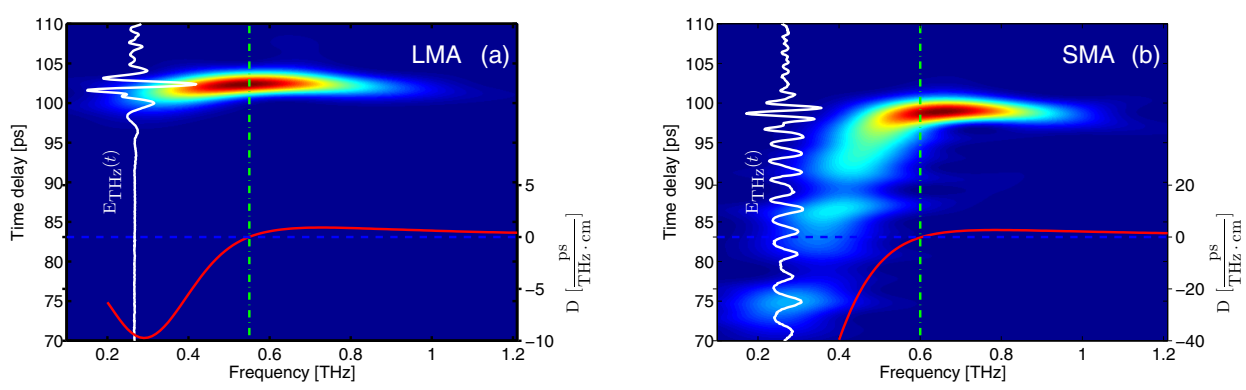

Fig. 2. Measured transmitted THz spectrogram for both fibers with corresponding numerically calculated dispersion. In both (a) and (b) the white trace is the transmitted THz signal through (a) $29 \mathrm{~mm}$ of LMA fiber and (b) $26 \mathrm{~mm}$ of SMA fiber, in arbitrary units. The colored image is a spectrogram generated by using a 3 ps wide Gaussian gating window. The red trace is the numerically calculated dispersion of the fibers.

The measured transmission of a single cycle THz pulse (with $2 \mathrm{THz}$ bandwith) through $29 \mathrm{~mm}$ of LMA fiber and $26 \mathrm{~mm}$ of SMA fiber are shown in Fig. 2 (a) and (b), respectively. In the figure the spectrogram of the pulse is also shown. This spectrogram is generated by using the experimental transmission data and gating it with a 3 ps Gaussian window. At each gating step a FFT is made and the frequency components of the pulse at that step are found. The spectrogram is generated to illustrate the dispersion of the two fibers. From the transmitted signal, shown as a white trace in Fig. 2 (b), together with the spectrogram it is clear that the SMA fiber has significant normal dispersion at least 4 times larger than the LMA fiber. This is to be expected since the core of the fiber is very small. The calculated dispersion of each fiber is shown as a red trace at the bottom of the figures. The dispersion is defined as: $\mathrm{D}=\frac{1}{2 \pi} \frac{\partial^{2} \beta}{\partial \nu^{2}}$ where $\beta$ is the propagation constant and $\nu$ is the frequency. The dispersion as shown here has the unit $\left[\frac{\mathrm{ps}}{\mathrm{THz} \cdot \mathrm{cm}}\right]$ meaning that the dispersion is the temporal broadening as a function of the bandwith of the THz pulse and length of fiber. The propagation constant can be numerically calculated and thereby the dispersion can also be calculated. These calculations can be compared to the spectrogram and reveal that the measured THz transmission fits nicely with the numerically calculated dispersion found using COMSOL. In both fibers most of the pulse is located at the zero dispersion frequency. The rest of the pulse is chirped, most significantly by the normal dispersion and only a little by the anomalous dispersion. The effects of the normal dispersion are clearest in the SMA fiber.

Cutback measurements were made on the LMA fiber. From these measurements the broadband loss was found. The broadband loss is defined as the total attenuation of the sum of the electric field squared $\left(\sum|\mathrm{E}(\mathrm{t})|^{2}\right)$. The Broadband loss is found to be $\alpha_{\mathrm{dB}}=0.4 \pm 0.06 \mathrm{~dB} / \mathrm{cm}$ corresponding to a absorption coefficient of $\alpha=0.09 \mathrm{~cm}^{-1}$. This value is lower than the measured bulk loss of the Topas of $\alpha=0.15 \mathrm{~cm}^{-1}$. This is to be expected because the propagation loss is determined by the overlap between the guided mode and the material. In a PCF much of the guided mode is overlapping with the air inside the holes. This overlap has practically zero loss. The loss of the fiber will be the bulk loss minus the overlap between the guided mode and the holes of the structure. Therefore the propagation loss will be less than the bulk loss.

In conclusion, we have fabricated Topas THz PCFs that are robust and have low loss. The fibers can be bent by heating and transmission through tight bends is possible. For the first time the mode profile of the fibers has been measured in the near field using electro-optic sampling. The broadband loss of the LMA fiber has been measured using cutback measurements.

\section{References}

1. A. Sengupta, A. Bandyopadhay, B. F. Bowden, J. A. Harrington, and J. F. Federici, "Characterisation of olefin copolymers using terahertz spectroscopy," Electronics Letters 42, 1477 (2006)

2. A. J. L. Adam, J. M. Brok, M. A. Seo, K. J. Ahn, D. S. Kim, J. H. Kang, Q. H. Park, M. Nagel, and P. C. M. Planken, ”Advanced terahertz electric near-field measurements at sub-wavelength diameter metallic apertures," Opt. Express 16, 7407 (2008)

3. G. Emiliyanov, J. B. Jensen, O. Bang, P. E. Hoiby, L. H. Pedersen, E. M. Kjaer, and L. Lindvold, "Localized biosensing with Topas microstructured polymer optical fiber," Optics Letters 32, 460-462 (2007) 